Received: January 23, 2017 Accepted: Feburary 03, 2017 Published: February 09, 2017

\section{A Staged Feasibility Study of a Novel Vaginal Bowel Control System for the Treatment of Accidental Bowel Leakage in Adult Women}

\author{
Michelle Miki Takase-Sanchez ${ }^{1, *}$ \\ ${ }^{1}$ Ventura County OB/GYN, 2949 Loma Vista Rd, Ventura, CA 93003-1544.
}

*Corresponding author: Michelle Miki Takase-Sanchez, MD, MS, FACOG, Ventura County Ob/Gyn, 2949 Loma vista Rd, Ventura, CA 93003-1544, Fax: (805) 643-2087;Tel: 805-6438695;E-mail: mmtsanchez88@gmail.com

\section{Abstract}

\subsection{Background}

Accidental bowel leakage, or fecal incontinence, impacts the quality of life in women of all ages. A minimallyinvasive vaginal bowel control system was designed to reduce accidents and provides a new health care option for women.

\subsection{Methods}

A feasibility study was conducted to evaluate fit, patient comfort, and ease-of-use of this novel vaginal bowel control therapy at home to better inform device design, treatment delivery, and the design of a subsequent pivotal clinical trial protocol. Staged evaluations were performed in women without and with self-reported accidental bowel leakage of any severity. Wear duration progressed from an initial one-time, in-office fitting to extended-wear periods at home. Device-related adverse events were collected in all subjects exposed to the device. Treatment responses were collected at baseline and after 1-month wear in women with accidental bowel leakage. Additionally, device comfort and satisfaction were assessed.

\subsection{Results}

Eighty-six women were fitted with forty-five women continuing to wear the vaginal bowel control system for $\geq 1$ week. Fifteen women with fecal incontinence were extended to $\geq 1$-month wear. Nine minor devicerelated adverse events were reported. Eight of 9 women who completed diaries experienced $\geq 50 \%$ reduction in episodes at 1-month wear. Device comfort and satisfaction were high.

\subsection{Conclusions}

This progressively staged, clinical evaluation study demonstrated the feasibility of extended wear of a novel vaginal bowel control system for the treatment of accidental bowel leakage. Positive response endpoints at 1-month were observed along with a good safety profile and high device satisfaction. These findings informed subsequent clinical delivery and trial design.

\section{Keywords}

Accidental bowel leakage, Bowel Control, Feasibility, Fecal Incontinence, Vaginal Insert

\section{Introduction}

Accidental bowel leakage (ABL), the patient-preferred term for fecal incontinence (FI) affects $12-15 \%$ of community-dwelling women [1-5]. Recent community-based studies estimate that as many as 17 million women in the U.S. suffer from some form of ABL, with over 3 million severe cases [6]. ABL prevalence is expected to increase as the population of the Unites States continues to age [5-7]. The condition is both physically limiting and emotionally devastating $[8,9]$ and represents a significant and growing unmet need in women's health.

Treatments for ABL range from conservative to invasive options [10]. Current non-surgical treatment options that include behavioral and dietary modifications, physical therapy, biofeedback, and medications often fall short of achieving adequate relief from ABL episodes [11, 12]. Additionally, surgical options are challenged with cost, medical and surgical complexity, and sub-optimal overall treatment success rates[2, 13-17]. Alternative treatments that provide long-term efficacy with minimal risk are needed.

Richter et al, recently described the pivotal study of a lowrisk, minimally-invasive, and effective treatment for fecal incon- 
tinence in women[18]. This novel vaginal bowel control (VBC) system (Eclipse ${ }^{\mathrm{TM}}$ System, Pelvalon, Inc. Sunnyvale, CA, USA) consists of a vaginal insert and user-controlled pressure-regulated pump (Figure 1). The vaginal insert consists of a silicone-coated stainless steel base and posterior directed balloon that provides reversible occlusion of the rectum that enables the user to regain control of her bowel function (Figure 2).

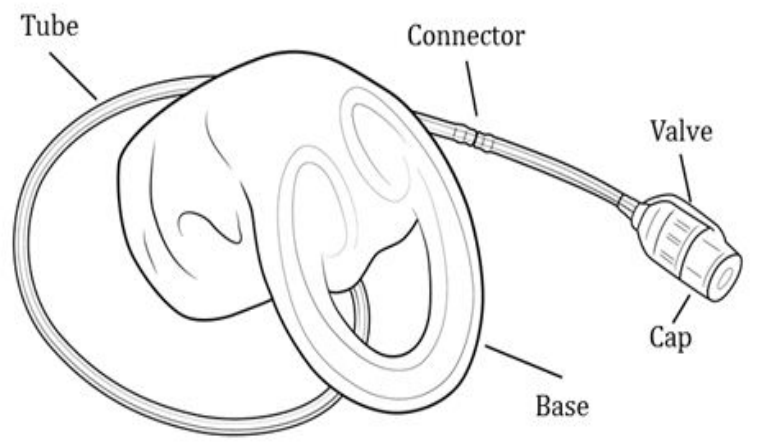

Figure 1: Vaginal Bowel Control (VBC) insert (printed with permission from (C) Julia Stack).
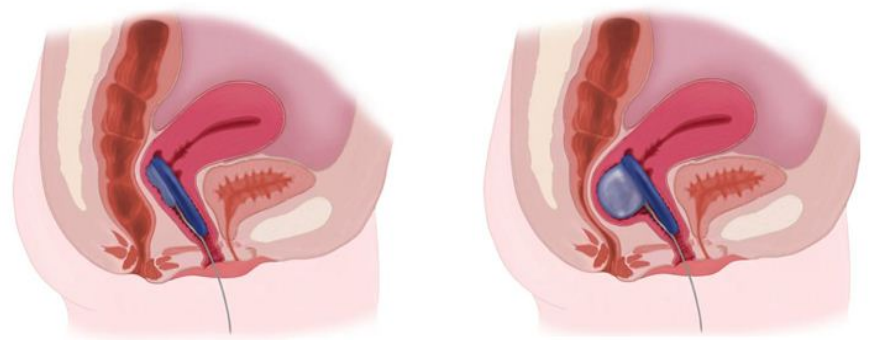

Figure 2: Vaginal Bowel Control (VBC) insert deflated (left) and inflated (right) (courtesy of Pelvalon).

Prior to that pivotal study, research was conducted to understand the feasibility of the concept of a vaginal bowel control system. Medical device development involves multiple stages of evolution driven by patient and clinician feedback derived from investigational trial use and standard care delivery [19-21]. Feasibility studies are intended to provide initial information about the safety, effectiveness, and acceptance by both patients and clinicians of a new therapy in a small, sample population. Flexibility in trial design allows the researchers to progress the therapy evaluations in response to incremental findings. The aim of this feasibility study was to observe patient usage of and satisfaction with the insert initially in an office-setting and eventually over increasingly long periods of wear at home. Secondary aims included the evaluation of data collection tools, and clinical protocol design.

\section{Materials and Methods}

Institutional review board (IRB) approval was obtained from Schulman Associates IRB (Schulman Associates, Inc. Cincinnati, $\mathrm{OH}, \mathrm{USA}$ ). All participants provided written informed consent for this prospective, open-label clinical study in adult women. All women age $>18$ years with and without fecal incontinence who were able and willing to provide written informed consent in English were eligible for participation. Exclusion criteria included the following: presence of a genitourinary or recto vaginal fistula, tumor, infectious process, an astomosis, or open wound; current or anticipated pregnancy; and any significant medical condition or other factor per investigator discretion that would interfere with study participation and/or increase participant risk.

Demographic data collected included age, race, menopausal status, and history of hysterectomy. Device data collected included device base size and overall shape, balloon size, and pump pressure. Clinical data collected included the following: clinician feedback on the value of various metrics of device fitting and stability; duration of device wear period (days, weeks, months); treatment response by bowel diary and/or ABL questionnaire; patient feedback on device comfort and satisfaction; and adverse events.

Device base sizes ranged from 44-88 $\mathrm{mm}$ lengths with various combinations of widths. Shapes included taper, round and elliptical. Balloon sizes varied from $32-47 \mathrm{~mm}$ depth with various combinations of widths. Pressure regulators included 5 different, non-overlapping, discrete ranges of balloon pressures.

The series of staged, clinical evaluations began first with recruitment of non-ABL and ABL participants with a limited inoffice only assessment with the intention of determining and understanding various metrics that constitute a good fitting. Participants in this phase of the study are included in the Safety Cohort. Clinician and participant feedback on each device fitting, with some subjects experiencing more than one fitting with different device types, was utilized in an effort to optimize device safety, stability and comfort, and to confirm the appropriateness of design changes. In the next stage of enrollment, participants who enrolled in the study joined the Short-Term Cohort, and wore the insert for one week to evaluate comfort and effect on the vaginal tissue. No quantitative measures of device effectiveness or satisfaction were captured for patients in the Short-Term Cohort or who were only included in the limited in-office only assessment, although demographic and safety data were collected as described below.

A sample of participants with ABL were then invited to join the Extended-Wear Cohort, extending their wear period for a duration of 1 month and then 3 months. This convenience sample of patients was selected based on the timing of the patients' enrollment in the study, their own preference, and their ability to demonstrate the ability to self-manage insertion and removal of the VBC system.

A baseline ABL history questionnaire was completed by the Extended Wear participants with fecal incontinence, and later in the study, a baseline bowel diary was introduced for newly enrolled patients. Follow-up assessments via a device satisfaction questionnaire were collected at 1-month and 3-month endpoints; at a midpoint in the study, questions assessing comfort were added into this questionnaire (comfort was previously assessed verbally and not documented on a consistent scale). For subjects enrolled later in the study, a repeat bowel diary was also 
collected at 1 month. Subjects who filled out a bowel diary were asked to document a 2-week period of daily frequency of soiling, stool consistency (Bristol Stool Scale) and severity (staining, minor or major soiling) and any associated fecal urgency episodes. Minor episodes were defined as soiling that did not require an immediate change of undergarment, pad, or clothing and major episodes were defined as soiling that required an immediate change of undergarment, pad, or clothing. Margin of treatment effectiveness was calculated as the percent reduction in the total number of minor and/or major episodes from baseline to treatment diaries.

Concurrent strict safety surveillance was conducted throughout the study for all Cohorts. Participants were provided a 24-hour/7-day hot line to report any symptoms of discomfort or concerns. Adverse events (AEs) were collected in all subjects exposed to the device. An AE was defined as any undesirable medical occurrence in a subject regardless whether a causal relationship with the device was determined. The investigator assessed each event for a possible relationship to the study device using the following definitions: Not related - not associated with device application and/or due to an underlying or concurrent illness or effect of another device or drug; Unlikely - little or no temporal relationship to the study device and/or a more likely alternative etiology exists; Possible - temporal sequence between device application and event is such that the relationship is not unlikely; Probable - temporal sequence is relevant or event abates upon device removal; Highly Probable - temporal sequence is relevant and event abates upon device removal or reappearance of the event on repeat device re-application. AEs were described as mild (transient and easily tolerated by the patient), moderate (causes the patient discomfort and interrupts her usual activities), or severe (causes considerable interference with the patient's usual activities; may be incapacitating and may require hospitalization).

\section{Results}

Study enrollment began in September 2011 and ended June 2013. One hundred twelve women were consented for participation. A total of 86 women (42 non-ABL, $44 \mathrm{ABL}$ ) were fitted with the device (the Safety Cohort). These women had a mean age of 52 (range 19-86 years) and were predominantly Non-Hispanic White, post-menopausal, and did not report a prior hysterectomy. Of the 44 subjects who reported bothersome ABL history, 42 completed a baseline ABL symptom questionnaire (2 women did not complete the questionnaire). Of these 42 subjects, 9 (21\%) reported having symptoms for less than 1 year, 18 (43\%) had symptoms for 1-5 years, 8 (19\%) had symptoms for 5-10 years, and 7 (17\%) had symptoms for over 10 years.

Clinical data results were collected from all 86 women who were fitted. The length of wear periods varied from 1 day (inoffice fitting only) to 101 days. For women who participated in the in-office visit only, the fitting clinician's observation of fit and the subject's feedback on device comfort was captured to develop recommendations on device fitting procedure. Forty-five women, including women with ABL as well as healthy volunteers, were then invited to participate in the Short-Term Cohort and contin- ued wear for one week. These subjects returned after one week to provide feedback on insert comfort and to undergo a pelvic exam to assess vaginal tissue.

After reasonable assurance of device comfort and safety was achieved through one-week wear periods, 15 women with ABL of any severity were invited to join the Extended-Wear Cohort and continue for a longer period of 1 month. Seven of these 15 women with ABL were subsequently extended to $\geq 3$-months' duration. Baseline and 1-month assessments by questionnaires were available for 14 of the 15 participants, with matched baseline and 1-month bowel diaries completed by the final 9 participants enrolled in the study.

The questionnaires completed by 14 participants after 1 month of insert usage included assessments of device satisfaction. Thirteen subjects reported they would continue using the VBC insert after study completion. All 14 subjects (100\%) reported they would recommend the device to a friend.

Of the 7 subjects who were asked to report on comfort on a documented assessment form, all women responded and the majority $(6 / 7,86 \%)$ reported, "I can't feel the device" and the other subject reported she could "feel the device but [it was] not uncomfortable." There were no participants who discontinued use of the trial device due to device dissatisfaction. Of the 9 women who kept bowel diaries; each experienced a minimum of one ABL episode over a 2-week baseline period. Eight of the 9 subjects (89\%) experienced $\geq 50 \%$ reduction in episodes, with 5 of these women experiencing a $100 \%$ reduction. A summary of these findings is presented in (Figure 3).

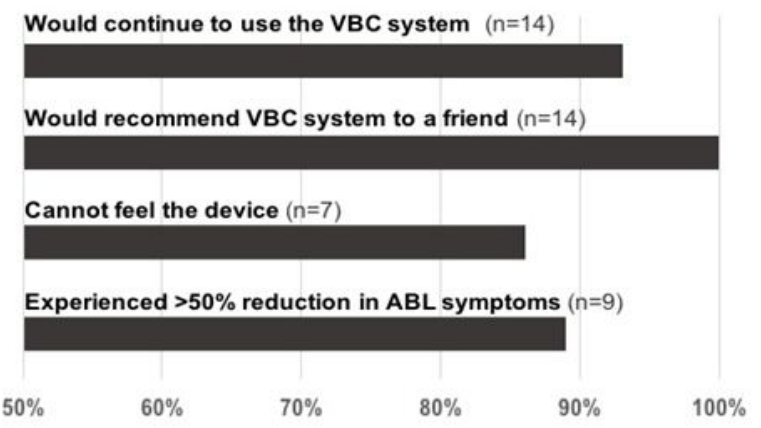

Figure 3: Assessments of device satisfaction, comfort, and efficacy in Extended Cohort following 1-month duration of daily wear. Baseline and 1-month assessments by questionnaires were available for 14 of the 15 participants. As this feasibility study also evaluated data collection instruments, matched baseline and 1-month bowel diaries were completed by the final 9 participants enrolled in the study. Formal questions on device comfort were added to the questionnaire for the final 7 participants of the Extended Cohort

Among the Safety Cohort, a total86 subjects exposed to the device across all wear periods, there were 10 device-related AEs. All 10 events were described as mild. Six were minor vaginal abrasions or ecchymoses, 3 were minimal vaginal bleeding (spotting), and 1 patient experienced cramping during the fitting period. All 10 device-related AEs were transient and resolved com- 
pletely. These events were more commonly associated with the initial fitting period $(8 / 10)$. Of note, women who demonstrated signs and/or symptoms of vaginal atrophy during or after the initial fitting at any point in the study participation were provided vaginal estrogen therapy or a non-hormonal alternative. There were no participants who discontinued use of the device due to an ongoing device-related AE.

\section{Discussion}

This progressively staged, clinical evaluation study demonstrated the feasibility of a novel, intra-vaginal, bowel-control device system for the treatment of ABL in adult women. By studying the device in a staged series of assessments, investigators first demonstrated device safety and comfort with limited risk to the participant, prior to commencement of extended durations of device exposure in order to determine treatment efficacy. These evaluations also provided insights furthering the evolution of device design. Additionally, these longer duration periods of patient usage provided an initial signal of patient compliance and satisfaction, and willingness to use a removable vaginal insert in order to achieve bowel control. Finally, this study evaluated several data collection tools to assess VBC system satisfaction and ABL symptom relief, establishing the adoption of patient bowel diaries for subsequent clinical trials [18, 22].

The benefits of staged and incremental device design that includes clinicians, patients and manufacturers have been previously described in several recent studies. Medina et al. (2013) provided a comprehensive description of a medical device development process model, highlighting the multi-staged approach to design evaluation, resolution of user concerns, and adaptive actions in device design and delivery[21]. Blanford et al. (2014) noted that challenges in device usability are minimized before market introduction to patients when incorporating the concept of "work as done" rather than "work as imagined" models of medical device design (fitness for purpose) [19] . In a case study reported by Furniss et al. (2015) on the usability of a glucometer, the authors noted that feedback and insight that impacted incremental and non-incremental design decisions came from multiple sources, including traditional verbal reports by users, as well as observations of users [20].

This study similarly illustrated the value of an interdisciplinary approach to medical device development. It also demonstrated that safety and therapeutic efficacy could be systematically evaluated and optimized by incorporating patient and clinician insight on practical usability. Device data collected, which assisted and confirmed changes to device design throughout the study, were derived primarily from collaborative discussions between design engineers and clinicians, and with considerable weight on participant feedback regarding stability and comfort. All these changes were conducted with concurrent safety surveillance and were performed with the intent to validate overall fit of the insert, durability of the balloon and functionality of the pump.

Arain et al. defines feasibility studies as "pieces of research done before a main study" can be designed and conducted[23]. Feasibility studies in themselves do not necessarily have a primary outcome or require statistical analysis. Some important method- ological components of a feasibility study include the following: estimation on subject eligibility (appropriateness of inclusion and exclusion criteria); time scale of outcome measures (duration of intervention exposure required for a measurable outcome); details on follow-up (response rates and adherence); acceptability of the intervention (willingness of clinicians to recruit participants and of participants to be randomized); and preliminary hypothesis testing (proof of concept and clinically significant safety and efficacy end-points). These components ultimately provide a basis for designing the main study from which more robust data will determine the statistically significant outcome(s).

In the case of this novel bowel control system, this feasibility study laid the groundwork for the following pivotal study conducted by Richter et al [18]. In this multi center, prospectively designed study of 61 women who wore the insert for a minimum of one month, the primary endpoint was derived from twoweek bowel diaries. Patient feedback and questions regarding the bowel diary used for the feasibility study allowed for clarification of instructions in the instrument used in the pivotal study. Additionally, the usage of device satisfaction questionnaires in this feasibility study demonstrated the value of capturing device-specific satisfaction metrics and feedback from patients, in addition to validated scores and instruments. This feasibility study was enrolled prior to the initiation of the pivotal study, and none of the patients who participated in this study were enrolled in the subsequent pivotal study. Although this feasibility study is limited by the small number of women participating in the Extended-Wear Cohort, these early safety, effectiveness and satisfaction results were a close predictor of the results later found by Richter et al, in their patient population at one and three months.

In conclusion, this feasibility study described the step-wise, staged approach of a series of clinical evaluations that reported on device design and safety, patient usage at home, user comfort and satisfaction, and therapeutic efficacy. Most importantly, this study highlighted the positive effectiveness signal and sensitivity of a 1 month end-point of a novel bowel control system for the treatment of fecal incontinence in adult women. These results supported the need for and design of subsequent clinical trials [18].

In addition to the already complete LIFE study by Richter et al, future directions include continued clinical investigations of a larger cohort of women with fecal incontinence with a longer duration of wear to evaluate its long-term safety and effectiveness. A multi-center, prospective, open-label, 1-year outcome clinical study is currently underway (clinicaltrials.gov ID NCT02428595 LIBERATE Trial) [24].

\section{Funding Acknowledgements}

Study supported by an industry-sponsored grant from Pelvalon, Inc.

\section{References}

1. Bharucha AE, Dunivan G, Goode PS, Lukacz ES,Markland 
AD,Mott L, et al. Epidemiology, pathophysiology, and classification of fecal incontinence. Am J Gastroenterol. 2015;110(1):127136.

2. Brown, SR, Wadhawan, H, and Nelson, RL. Surgery for faecal incontinence in adults.Cochrane Database Syst Rev. 2010;8(9):CD001757.

3. Meyer I, Richter HE. Impact of fecal incontinence and its treatment on quality of life in women.Womens Health (Lond Engl). 2015;11(2):225-238.

4. Nygaard I, Barber MD, Burgio KL, et al. Prevalence of symptomatic pelvic floor disorders in US women. JAMA. 2008;300(11):1311-1316.

5. Varma MG, Brown JS, Creasman JM,Thom DH,Subak LL,Beattie Ms, et al. Fecal incontinence in females older than aged 40 years: who is at risk? Dis Colon Rectum. 2006;49(6):841-851.

6. Wu JM, Vaughan CP, Goode PS,David TR,Holly ER,Burgio KL, et al. Prevalence and trends of symptomatic pelvic floor disorders in U.S. women. Obstet Gynecol. 2014;123(1):141-148.

7. Wu JM, Hundley AF, Fulto, RG,Myers ER. Forecasting the prevalence of pelvic floor disorders in U.S. Women: 2010 to 2050. Obstet Gynecol. 2009:114(6)1278-1283.

8. Bharucha, AE, Zinsmeister, AR, Locke, GR, Seide BM, Schleck CD,Melton LJ,et al. Prevalence and burden of fecal incontinence: a population-based study in women. Gastroenterology. 2005;129(1):42-49.

9. Markland AD, Greer WJ, Vogt A,Redden DT,Goode Ps,Burgio $\mathrm{Kl}$, et al. Factors impacting quality of life in women with fecal incontinence. Dis Colon Rectum. 2010;53(8):1148-1154.

10. Whitehead WE, Rao SS, Lowry A,Nagle D, Varma $\mathrm{M}$,Bitar $\mathrm{KN}$, et al. Treatment of fecal incontinence: state of the science summary for the National Institute of Diabetes and Digestive and Kidney Diseases workshop. Am J Gastroenterol. 2015;110(1):138-146

11. Heymen S, Scarlett Y, Jones K, Ringel Y,Drossman D, Whitehead WE. Randomized controlled trial shows biofeedback to be superior to pelvic floor exercises for fecal incontinence. Dis Colon Rectum. 2009;52(10): 1730-1737.

12. Norton C. Behavioral management of fecal incontinence in adults. Gastroenterology. 2004121(1 Suppl 1): S64-S70.

13. Bravo Gutierrez, A, Madoff, RD, Lowry, AC,Parker SC,Buie WD,Baxter NN. Long-term results of anterior sphincteroplasty.Dis
Colon Rectum. 2004;47(5):727-731.

14. Glasgow SC, Lowr, AC. Long-term outcomes of anal sphincter repair for fecal incontinence: a systematic review. Dis

Colon Rectum. 2012;55(4):482-490.

15. Hetzer FH, Bieler A, Hahnloser D,Lohlein F,Clavien PA,Demartiens N. Outcome and cost analysis of sacral nerve stimulation for faecal incontinence.Br J Surg. 2006;93(11):14111417.

16. Melenhorst J, Koch SM, Uludag O,VanGemert WG,Baeten CG. Sacral neuromodulation in patients with faecal incontinence: results of the first 100 permanent implantations. Colorectal Dis. 2007;9(8):725-730.

17. Wexner SD, Coller JA, Devroede G,Hull T,Chan M,Snape WJ, et al., Sacral nerve stimulation for fecal incontinence: results of a 120-patient prospective multicenter study.Ann Surg. 2010; 251(3): 441-449.

18. Richter HE, Matthews CA, Muir T,Hale DS,Van Drie D,Varma MG, et al., A vaginal bowel-control system for the treatment of fecal incontinence. Obstet Gynecol. 2015;125(3):540-547.

19. Blandford A, Furniss D, and Vincent C. Patient safety and interactive medical devices: Realigning work as imagined and work as done.Clin Risk. 2014;20(5):107-110.

20. Furniss D, Masci P, Curzon P,Astrid M,Blanford A. Exploring medical device design and use through layers of distributed cognition: how a glucometer is coupled with its context. J Biomed Inform. 2015;53:330-341.

21. Medina LA, Okudan Kremer GE, Wysk RA. Supporting medical device development: a standard product design process model. Journal of Engineering Design. 2013;24(2):83-119.

22. Varma MG, Matthews CA, Muir T,Holly ER,Hale DS,Van DD, et al. Impact of a novel vaginal bowel control system on bowel function. Dis Colon Rectum. 2016; 59(2):127-131.

23. Arain, M, Campbell, MJ, Cooper, CL,Gillian AL. What is a pilot or feasibility study? A review of current practice and editorial policy.BMC Med Res Methodol. 2010;10:67.

24. Pelvalon. A Clinical Evaluation of the Eclipse ${ }^{\mathrm{TM}}$ System, a Vaginal Bowel Control (VBC) Therapy for Fecal Incontinence in Women (LIBERATE). 